\title{
The Development of 'Senescence' in Podospora anserina
}

\author{
By J. R. SMITH AND I. RUBENSTEIN* \\ Department of Molecular Biophysics and Biochemistry, Yale University, \\ New Haven, Connecticut, 06520, U.S.A.
}

(Received 4 January 1973)

\begin{abstract}
SUMMARY
The growth of a mycelium of Podospora anserina ceases after a period of time characteristic for a given race. As the mycelium grows out from the germinated spore it passes through two physiological states; a 'non-senescent' state where the growth potential of the various regions of the growing mycelium remains constant and a 'senescent' state where the growth rate remains constant but the growth potential of the mycelium progressively decreases.

The 'senescent' state is apparently due to the presence of a variant cytoplasmic determinant which is discontinuously distributed among the cells of the mycelium and whose concentration increases exponentially during growth. The rate of transformation from the 'non-senescent' to 'senescent' state is directly proportional to the number of hyphae per mycelium. The median length of growth can be greatly extended by short periods of growth in the presence of cycloheximide or cyanide, but not by a variety of other drugs which inhibit mitochondrial functions.
\end{abstract}

\section{INTRODUCTION}

'Senescence' is a cytoplasmically inherited variant of Podospora anserina. The ultimate manifestation of 'senescence' is cessation of mycelial growth and eventual death. The 'senescent' phenotype develops in all mycelia of $P$. anserina after long periods of vegetative culture (Rizet, I953a). Marcou (I96I) introduced the idea that mycelia contained the determinant of 'senescence' (a 'senescence' factor) sometime before the actual stoppage of mycelial growth. The phenotypic expression of the early stages of 'senescence' is declining growth potential as growth proceeds (Marcou, I96I). The 'senescent' phenotype can be transmitted to as many as $90 \%$ of the progeny by a 'senescent' protoperithecial parent but to none of the progeny by a 'senescent' spermatial parent (Rizet, I957). Furthermore, the 'senescent' phenotype can be induced or transferred to a 'non-senescent' mycelium by hyphal anastomosis with a 'senescent' mycelium. Marcou \& Schecroun (I959) showed, using 'senescent' mycelium carrying a dominant nuclear marker, that nuclear transfer did not ordinarily occur during hyphal anastomosis, and concluded that the 'senescence' phenotype was caused by a cytoplasmic substance.

Some observations which have been made concerning 'senescence' may be summarized as follows: (i) Strains isolated from different geographical locations (termed geographical races or simply races) possess different characteristic median lengths of growth (M.L.G.). The M.L.G. is maintained in sexual crosses between cultures from the same geographical race (Rizet, $1953 b$ ). (ii) All the members of a 'non-senescent' mycelial population derived from a single race grow at least a certain distance that is race specific (Marcou, I96I),

* Present address: Department of Genetics and Cell Biology, University of Minnesota, St. Paul, Minnesota, 5510I, U.S.A. 
termed the incubation distance (I.D.). (For race S, I.D. $\approx 100 \mathrm{~cm}$; for race A, I.D. $\approx 10 \mathrm{~cm}$.) (iii) After reaching the amount of growth equal to the I.D., the number of surviving mycelia able to continue growth decreases exponentially with further growth (Marcou, 1961). (For race $\mathrm{S}$, the rate of decrease is about $0.01 / \mathrm{cm}$ of growth and for race $\mathrm{A}$, about $0.2 / \mathrm{cm}$.) The I.D. and the rate of decrease of surviving mycelia, termed the transformation rate (T.R.), are characteristic of a mycelial population and under most constant culture conditions depend only on the race from which the population was derived (Marcou, 196I). (iv) The values of M.L.G., I.D. and T.R. of a population of mycelia derived from interracial crosses between $\mathrm{S}$ and $\mathrm{A}$ are the same as those of the protoperithecial parent, regardless of the spermatial parent. There is no segregation of these characteristics among the progeny of an interracial cross (Smith \& Rubenstein, 1973). (v) If a mycelium is kept for long periods under conditions where growth is prevented then, at times, the reverse transformation from the 'senescent' to the 'non-senescent' state occurs (e.g. storage for I year at $3{ }^{\circ} \mathrm{C}$ ) (Marcou, 196I). (vi) A mycelium is transformed from the 'non-senescent' to the 'senescent' state by what appears to be a single random event (the appearance of a 'senescence' factor in the cytoplasm). The growth potential of a mycelium in the 'senescent' state decreases with additional growth (Marcou, I96r).

The experiments reported here were designed to contribute to our understanding of the events occurring during the development of the 'senescent' phenotype in Podospora anserina.

We will present evidence that: (i) Mycelia of race A, like race S, exist in two physiological states, and that a single random event is responsible for the transition from the first to the second state. (ii) The 'senescence' factor is particulate and the I.D. represents the amount of growth necessary for the number of 'senescence' factors to become sufficiently high to result in the expression of 'senescent' morphology. (iii) The T.R. from the 'non-senescent' to 'senescent' state is directly proportional to the number of hyphae per mycelium. (iv) The M.L.G. can be greatly extended by short periods of growth in the presence of cycloheximide or cyanide, but not by a variety of other drugs which inhibit mitochondrial function.

\section{METHODS}

Biological material. Stock mycelia of Podospora anserina races A and S were obtained from Dr D. Marcou, Orsay, France. The different races are distinguished by M.L.G., I.D., T.R., (Marcou, I96I) barrage reaction, (Rizet, Marcou \& Schecroun, I958; Schecroun, 1962; Bernet, I965; Esser \& Kuenen, 1967) and semi-incompatibility reactions (Esser \& Kuenen, 1967). These original mycelial stocks and all subsequent stocks were stored at $3{ }^{\circ} \mathrm{C}$ on corn meal agar slants.

Standard culture tubes. Ten $\mathrm{ml}$ of corn meal agar (Difco) were dispensed into $20 \times 150 \mathrm{~mm}$ glass tubes and the tubes stoppered with Teflon-covered, slotted corks. The slotted corks permit sufficient aeration. After sterilizing for $\mathrm{I} 5 \mathrm{~min}$ at $\mathrm{I} 2 \mathrm{I}{ }^{\circ} \mathrm{C}$ the tubes were laid horizontally and the agar allowed to harden. This created a partial solid cylinder of nutrient agar of constant cross-sectional area. To make our study of 'senescence' as quantitative as possible we considered it important to keep the number of hyphal tips/tube constant throughout the growth of the mycelium.

Transfer procedure. The culture tubes were inoculated with a small piece of mycelium or a germinated ascospore near the open end of the tube. Growth occurred toward the closed end of the tube. In view of the finding of Rizet et al. (1958) that the size and frequency of transfer had an influence on M.L.G., we attempted to develop a transfer procedure which approached as closely as possible the situation obtained in continuous growth tubes. The 


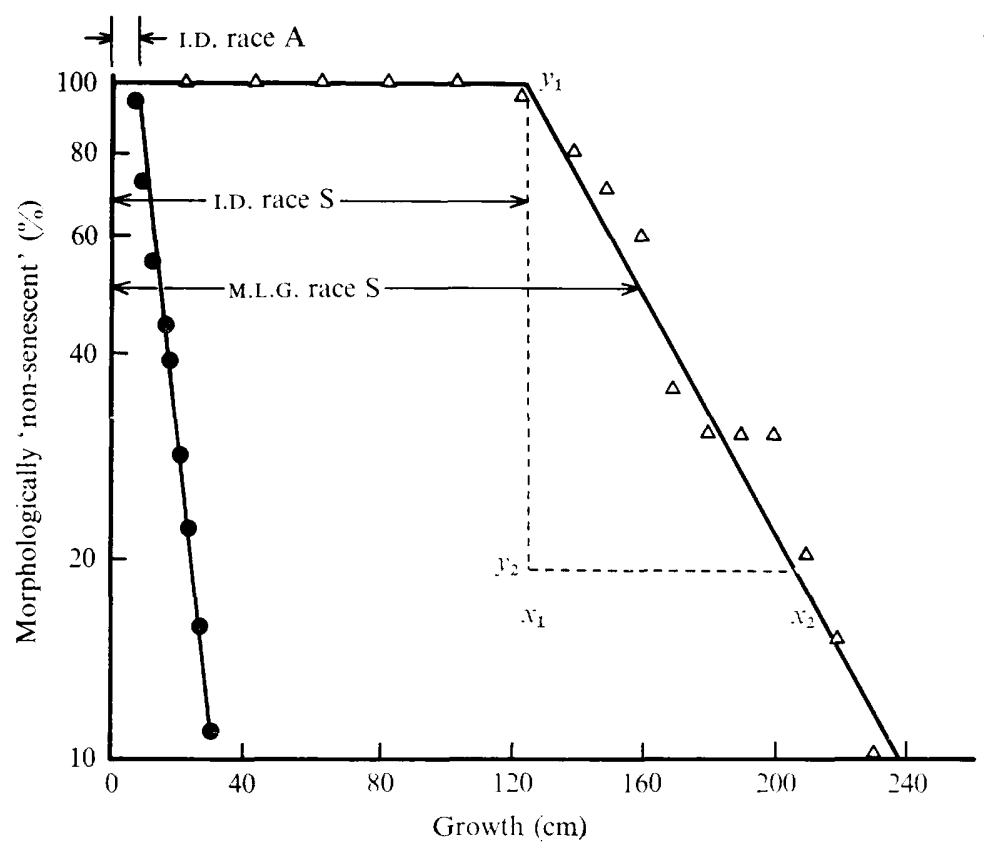

Fig. I. The percentage of the mycelial populations of race $\mathrm{A}$ and race $\mathrm{S}$ morphologically 'nonsenescent' is plotted as a function of distance grown. Each mycelial population was composed of 20 mycelia each originating from a binucleate ascospore and cultured in standard culture tubes on corn meal agar at $26{ }^{\circ} \mathrm{C}$ without illumination (standard culture conditions). M.L.G., median length of growth; I.D., incubation distance; T.R., transformation rate $=\left(\log y_{1}-\log y_{2}\right) /\left(x_{2}-x_{1}\right)$. , race $\mathrm{A} ; \triangle$, race $\mathrm{S}$.

mycelium was cut $\mathrm{I} \mathrm{cm}$ behind the growing edge and removed with a sterile stainless steel tool. The agar was then cut just ahead of the growing front and the last $\mathrm{I} \mathrm{cm}$ of mycelial growth was transferred to a fresh tube. A $\mathrm{r} \mathrm{cm}$ section of nutrient agar was removed from the fresh tube to accommodate the transfer. Using this method of transfer, none of the hyphae near the growth point were injured and there was no discontinuity in the growth rate. Unless stated otherwise, all cultures were grown at $26 \pm \mathrm{I}{ }^{\circ} \mathrm{C}$ and transferred every I5 days.

Median length of growth determination. The M.L.G. was determined for populations of mycelia in which each mycelium resulted from the germination of a single ascospore and also for populations of mycelia which resulted from subcultivation of a single mycelium. To determine the M.L.G. for the mycelia from a spore population, the spores were germinated (usually 20 to 50 from a given cross) and each young mycelium transferred to a culture tube about $20 \mathrm{~h}$ after germination (the mycelia are less than $\mathrm{I} \mathrm{mm}$ in diam. at this time). The total distance grown by each mycelium was noted after growth ceased, and the M.L.G. determined. The T.R. and I.D. can also be determined for a spore population from this information (Fig. I).

In some cases it was desirable to obtain the M.L.G. of samples taken from a small region of a single mycelium. This was accomplished by subculturing Io transverse slices ( $\mathrm{mm}$ thick) cut from a mycelium growing in a standard culture tube. Each slice contained the entire cross-section of the mycelium. The M.L.G. of these subcultured mycelia was taken to be the M.L.G. of the original mycelium. 
Table I. Median length of growth, transformation rate and incubation distance of races $A$ and $S$

The values of these three parameters were obtained from ten populations of mycelia of at least 25 members each; the standard deviations are indicated.

$\begin{array}{lccc}\text { Mycelia } & \text { M.L.G.* }(\mathrm{cm}) & \text { T.R.* }\left(\mathrm{cm}^{-1}\right) & \text { I.D.* }(\mathrm{cm}) \\ \text { Race A } & 15 \pm 2 & 0.2 \pm 0 . \mathrm{I} & 10 \pm \mathrm{I} \\ \text { Race S } & 170 \pm 40 & 0.013 \pm 0.005 & 100 \pm 30\end{array}$

* M.L.G., median length of growth; T.R., transformation rate; I.D., incubation distance.

Estimate of the number of hyphae per culture. The most direct method for determining the number of hyphae growing through a transverse cross-section of the culture is to examine such a cross-section microscopically and count the number of hyphal ends. Some difficulty arises because the hyphae do not grow exactly perpendicular to the transverse cross-section and because, in some media, the density of hyphae is not constant across the slice. Therefore, an alternative method was used to determine the hyphal density. A $5 \mathrm{~mm}$ thick transverse slice of mycelium was fragmented in a ground glass tissue homogenizer and the average hyphal length per fragment and the number of fragments per unit volume were determined. Their product was used to estimate the average hyphal density. This estimate will be too high because hyphal growth is not parallel as implied in the above calculation. Since only the relative hyphal density is important in this discussion and the patterns of growth are not noticeably different in the various media, this error is not critical. Repeated measurements of hyphal density agreed within $\pm 10 \%$.

\section{EXPERIMENTAL}

\section{Existence of 'senescent' and 'non-senescent' states of a mycelium}

When a group of samples taken from a mycelium several centimetres before 'senescent' morphology is expressed are subcultured, the resulting mycelia grow various distances before growth ceases. A curve analogous to a survival curve can be obtained by plotting as a function of distance the percentage of morphologically 'non-senescent' mycelia. Curves of this type are shown for races $\mathrm{A}$ and $\mathrm{S}$ in Fig. I where the percentage of the mycelia showing 'non-senescent' morphology is plotted as a function of distance of growth. These curves have two notable characteristics; I00 \% of the cultures grow a distance termed the I.D. before exhibiting 'senescent' morphology and thereafter the percentage of growing cultures decreases exponentially with growth. The rate of decrease is termed the T.R.

Marcou (196I) has interpreted the shape of this curve in the following manner. A mycelium can exist in two states; the 'non-senescent' and 'senescent' state. A mycelium in the 'non-senescent' state can be transformed to the 'senescent' state by a single random event (this event corresponds to the first appearance of a 'senescence' factor). The probability of an event occurring per unit growth has a constant value equal to the T.R. After the first appearance of the 'senescence' factor, an amount of growth equal to the I.D. is necessary before 'senescent' morphology is manifest (Fig. 2).

The same form for Fig. I would result if the transformation from 'non-senescent' to 'senescent' were the result of multiple events. This explanation was ruled out experimentally for race $S$ by Marcou (I96I).

The average values of M.L.G., T.R. and I.D. for races $A$ and $S$ derived from a number of different populations of mycelia are given in Table $\mathrm{I}$. 
Populations of mycelia derived from a small region of a mycelium of race $S$ that is 'senescent' can give rise to values for M.L.G., T.R. and I.D. approximately the same as those shown in Table I for race A. Therefore, it is necessary to determine whether the idea of 'non-senescent' and 'senescent' states is valid for race A, and to determine whether mycelia of race $\mathrm{A}$ behave as if a single random event were responsible for the transformation from 'non-senescent' to 'senescent' state. The alternative possibility is that 'non-senescent' mycelia of race $\mathrm{A}$ and race $\mathrm{S}$ have approximately the same values for M.L.G., T.R. and I.D. and that all the mycelia for race $\mathrm{A}$ used here and by Rizet and Marcou were in the 'senescent' state.

If mycelia of race A do exist in two physiological states then the M.L.G. of a group of subcultured mycelia derived from a small region of a growing mycelium will have a value nearly identical to another group taken from a different region as long as the groups of samples are taken from the 'non-senescent' portion of the mycelium. However, the M.L.G. of the groups of mycelia taken from the 'senescent' portion will depend on the region of the original mycelium from which they were derived. Samples taken near the point at which the mycelium became 'senescent' (i.e. soon after the appearance of the 'senescent' factor) will give rise to mycelia which grow farther than those taken after a few more centimeters of growth.

To test this idea a growing mycelium of race A originating from a germinated ascospore was sampled as growth proceeded. When sampling experiments of this kind are discussed the mycelium from which the samples were taken will be called the stock mycelium. When the stock mycelium had grown $2 \mathrm{~cm}$, the $\mathrm{I} \mathrm{cm}$ section of mycelium farther from the growing edge was removed and sliced transversely into Io slices, I mm in thickness. Each of these slices was subcultured in a separate culture tube. The $\mathrm{I} \mathrm{cm}$ section containing the growing edge was retained and its growth allowed to continue. The sampling was continued at $\mathrm{I} \mathrm{cm}$ intervals until the stock mycelium stopped growing. Each set of Io samples was used to obtain an estimate of the M.L.G., T.R. and I.D. for the $\mathrm{r} \mathrm{cm}$ region of stock mycelium from which it arose. After the stock mycelium and all the samples collected from it had stopped growing the data were collected and arranged relative to the position at which growth ceased in the stock mycelium. Fig. 3 shows the results of one such experiment. All the sets of samples taken more than $9 \mathrm{~cm}$ before the stock mycelium stopped growing have a M.L.G. distributed about $13 \mathrm{~cm}$. Those taken less than $9 \mathrm{~cm}$ away from growth stoppage show a linear decrease in M.L.G. as the samples were taken nearer the region where the stock mycelium stopped growing.

This experiment has been repeated using seven other mycelia of race A. In all cases the results were essentially the same as those shown in Fig. 3; the linear decrease in M.L.G. started between 9 and $13 \mathrm{~cm}$ from growth stoppage. The results of these experiments with race $\mathrm{A}$ are in agreement with Marcou's model. They indicate that mycelia of race A stop growing 9 to $13 \mathrm{~cm}$ after they enter the 'senescent' state.

The T.R. and the I.D. of these mycelial subcultures can be used as indicators of the change in the stock mycelium from the 'non-senescent' to 'senescent' state. The r.R. of subcultures from the 'non-senescent' portion of a stock mycelium should have a finite value indicative of the rate at which the individual mycelia enter the 'senescent' state. The T.R. of subcultures from the 'senescent' region of a stock mycelium should, ideally, be infinite. (In practice they are finite because of variations in the I.D.) Therefore, a discontinuity in the values of T.R. should occur at the point where the stock mycelium becomes 'senescent'. In addition, the values for the I.D. of subcultures from the 'non-senescent' region of a mycelium should be constant. But the I.D. of subcultures from the 'senescent' region should 


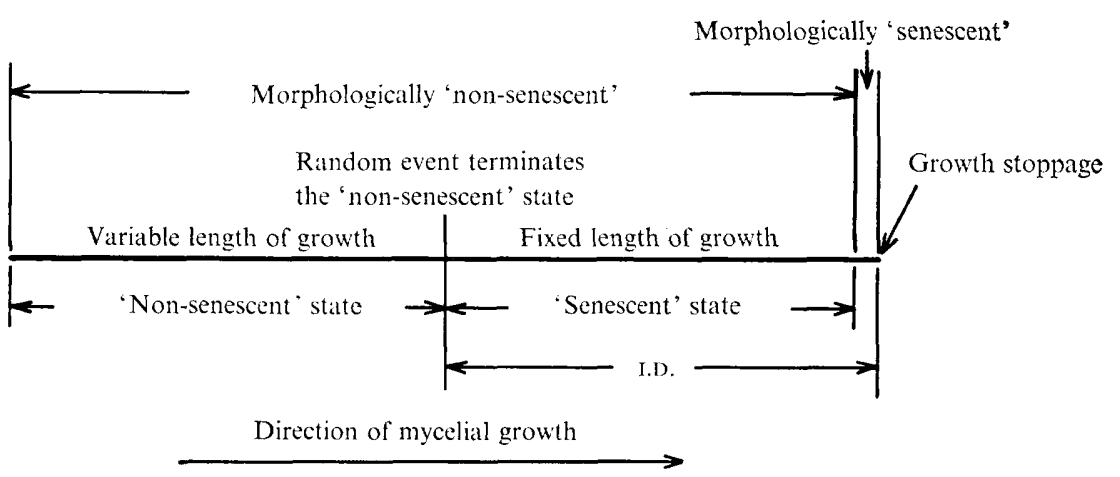

Fig. 2. Schematic representation of the two physiological and the two morphological states of a mycelium. I.D., incubation distance.

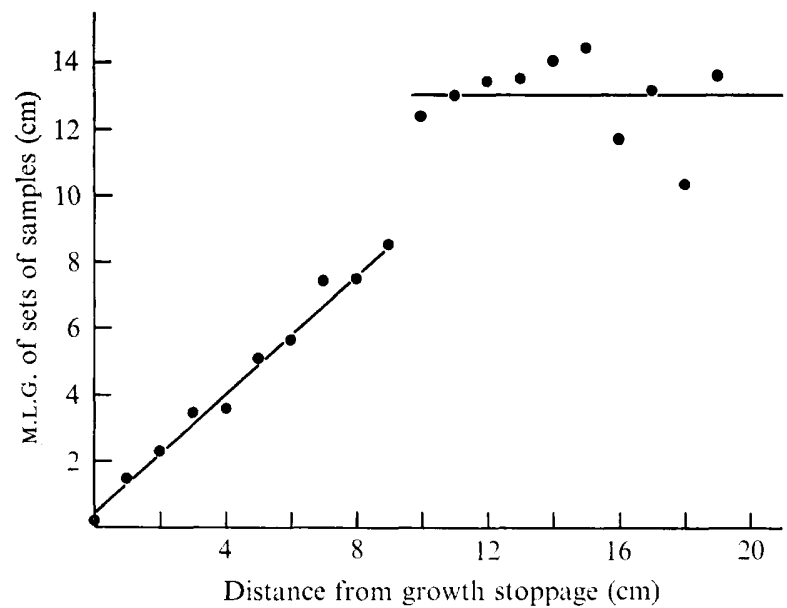

Fig. 3. The median length of growth of sets of mycelia as a function of distance from growth stoppage. The ten consecutive slices each of $0.1 \mathrm{~cm}$ thickness that represent a set were taken at the same time from the region of the mycelium between $I$ and $2 \mathrm{~cm}$ behind the growing edge of the stock mycelium.

depend on the position of sampling, becoming smaller as the stock mycelium advances further into the 'senescent' state.

Fig. 4 shows the I.D. and the T.R. derived from sets of samples as a function of distance from growth stoppage of the stock mycelium. This is the same stock mycelium for which the M.L.G. of the sets are plotted in Fig. 3. The I.D. varies between 8 and $\mathrm{II} \mathrm{cm}$ and the T.R. from 0.16 to $0.29 \mathrm{~cm}^{-1}$ in the 'non-senescent' region. The values for the I.D. and T.R. of the subcultures begin to change in the region of the stock mycelium which is 8 to $10 \mathrm{~cm}$ removed from where growth of the stock mycelium eventually ceased. The I.D. progressively declines and the T.R. increases almost stepwise to a maximum of about $1 \cdot 3 \mathrm{~cm}^{-1}$. For each of the stock mycelia tested the discontinuities in I.D. and T.R. occur in the same region of the stock mycelium as the decrease in M.L.G.

On the basis of the above observations, we are confident that the mycelia of race A can exist in two well defined states: 'non-senescent' and 'senescent'. The values of T.R. represent the rate at which the mycelia enter the 'senescent' state and the I.D. the amount of subsequent growth required for the morphological manifestation of 'senescence'. 


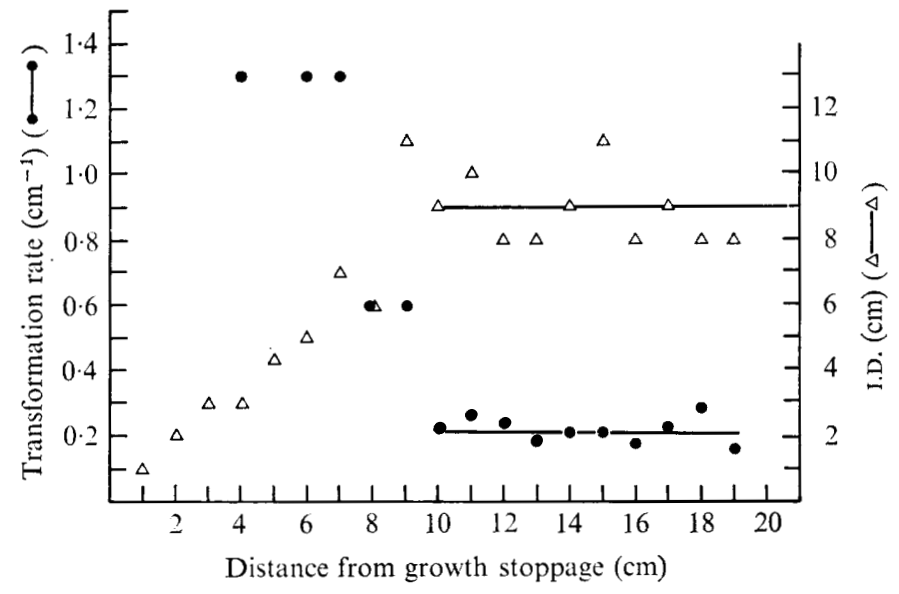

Fig. 4. Transformation rate $(----)$ and incubation distance $(\triangle---\triangle)$ of sets of mycelial samples as a function of position of sample origin for mycelia of race $A$. These values were derived from the same experiment as the values of M.L.G. shown in Fig. 3.

The increase in the number of 'senescence' factors in 'senescent' mycelia

Marcou (I96I) postulated that the concentration of the 'senescence' factor increased during growth of a mycelium in the 'senescent' state. The following experiments were performed to test this idea and to determine, roughly, the concentration of the 'senescence' factor.

Transverse slices were taken $\mathrm{I} \mathrm{cm}$ behind the growth front of a mycelium at various points during its growth. The slice was fragmented, the hyphal fragments diluted and plated on corn meal agar. After $24 \mathrm{~h}$, colonies that grew from these fragments were transferred to culture tubes ( 50 colonies for each slice). In every sample approximately $50 \%$ of the hyphal fragments were capable of forming mycelia. Each hyphal fragment contained an average of 20 cells, therefore each colony may be the result of the regeneration of $\mathrm{I}$ to 20 cells. If the 'senescence' factor is distributed discontinuously among the hyphal fragments (i.e. particulate), all hyphal fragments containing one or more 'senescence' factors will result in mycelia which grow only a short distance and those which contain no 'senescence' factors will grow a distance at least as great as the I.D. In addition, if the concentration of the 'senescent' factor increases with further growth the fraction of hyphal fragments containing one or more 'senescence' factors will increase as the slices are taken closer to the point where the mycelium ceases growth.

The distributions of length of growth among the mycelia resulting from the several sets of hyphal fragments are shown in Fig. 5. All the mycelia from samples taken more than 5.5 $\mathrm{cm}$ before growth stoppage occurred grew at least $7 \mathrm{~cm}$. As samples were taken closer to growth stoppage an increasing proportion grew less than $7 \mathrm{~cm}$.

If the assumption is made that the 'senescent' factors contained in any particular slice are free to move among the hyphae in that slice (this is reasonable since the hyphae are interconnected by frequent anastomoses), then the number of 'senescent' factors per hyphal fragment will follow a Poisson distribution. The probability of a given hyphal fragment containing $k$ 'senescence' factors is given by

$$
P(k)=\frac{\mathrm{e}^{-a}(a)^{k}}{k !}
$$



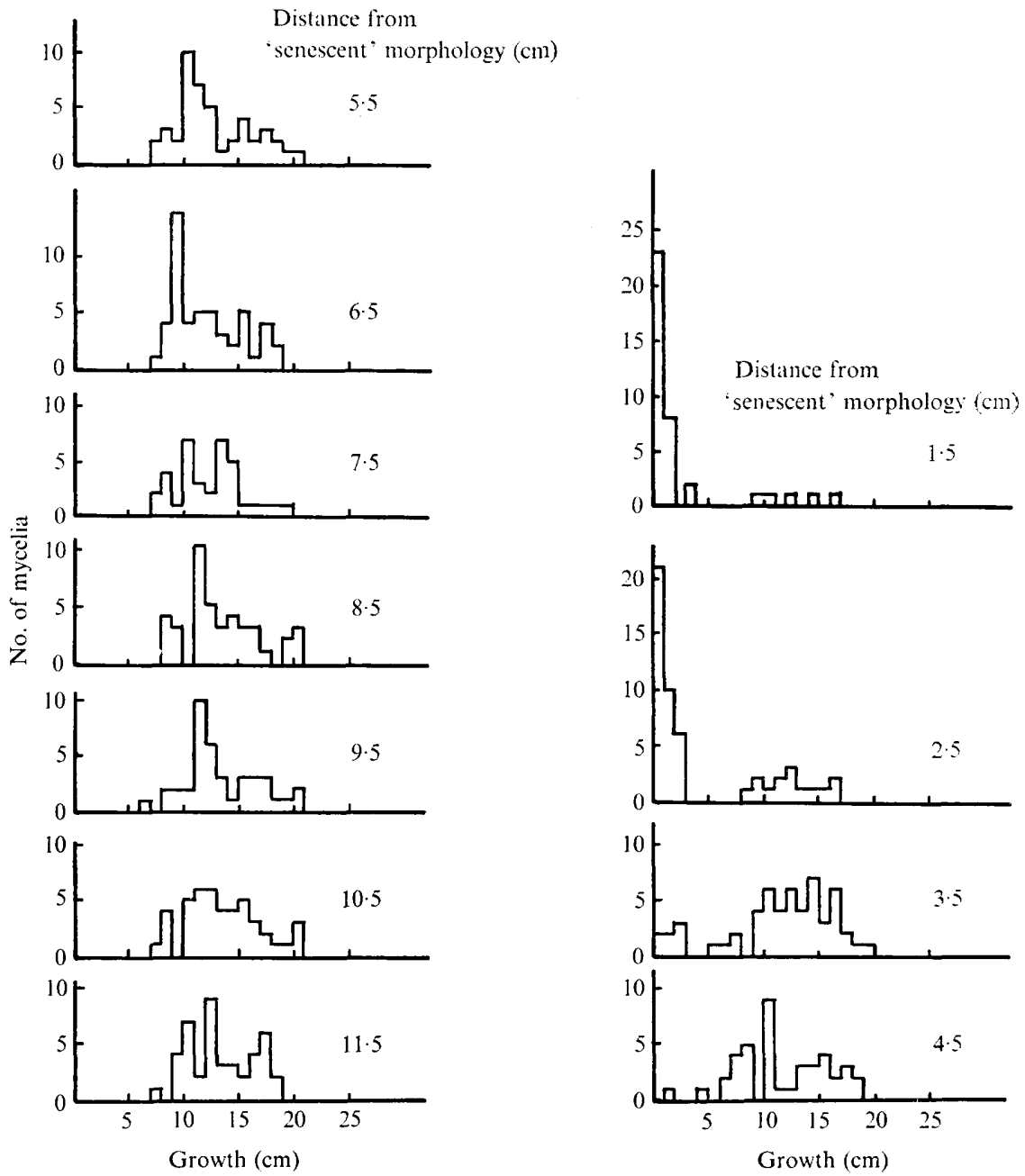

Fig. 5. Frequency distribution of the lengths of growth of mycelia from regenerating hyphal fragments. Each distribution represents about 50 mycelia which were obtained from hyphal fragments of a sample taken from a small region of the stock mycelium. The different distributions are from samples taken from various positions of the stock mycelium.

where $a$ is the average number of 'senescent' factors per hyphal fragment. If $P(0)$ the probability of a hyphal fragment having zero 'senescent' factors is known the value of $a$ can be calculated.

$$
a=-\ln P(0) \text {. }
$$

We used as $P(0)$ the proportion of viable hyphal fragments in a given sample which gave rise to mycelia growing at least $7 \mathrm{~cm}$. Fig. 6 is a semilogarithmic plot of $a$ versus distance to growth stoppage for three experiments of the type described above.

The idea was stated earlier that the I.D. corresponds to the amount of growth attained after the occurrence of one 'senescence' factor in a mycelium. As will be discussed later there are about $4 \times 10^{4}$ hyphal tips passing through a given cross-section of a mycelium growing under standard culture conditions. Each hyphal fragment used in this experiment 


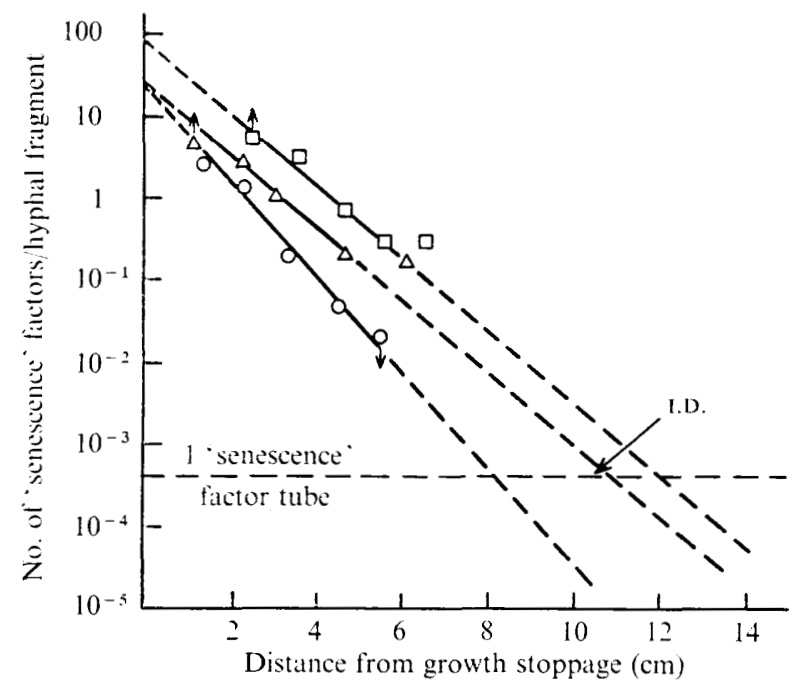

Fig. 6. Average number of 'senescence' factors per hyphal fragment in a mycelium of race A; $a$, plotted as a function of distance from the manifestation of 'senescent' morphology. The different symbols represent independent experiments performed on different mycelia of race $A(O, \triangle)$ or the interracial hybrid $\mathrm{A}\left(\mathrm{AS}_{10}\right)(\square)$ (Smith \& Rubenstein, I973).

contained an average of 20 cells. Therefore a given cross-section of mycelium contains an average of $2 \times 10^{3}$ hyphal fragments. Thus, one 'senescence' factor per mycelium corresponds to $5 \times 10^{-4}$ 'senescence' factors per hyphal fragment. By extrapolation of the data in Fig. 6 to this value an estimate of the I.D. can be obtained. These values range from 8 to $12 \mathrm{~cm}$ and are in good agreement with the values found in the proceeding section. By extrapolation to the point of growth stoppage, an estimate of 20 to 100 'senescence' factors per hyphal fragment was obtained. Since there are an average of 20 cells per hyphal fragment, this corresponds to an average of I to 5 'senescence' factors per cell. This estimate is probably too low since not all the cells of the fragmented hyphae appear to be viable. Some of them have lost their cytoplasm due to fragmentation. These results indicate, however, that the number of 'senescence' factors per cell in the region of morphological 'senescence' is probably not greater than 100 .

The 'senescence' factor, therefore, appears to be particulate and to increase exponentially in number with growth. The hypothesis that the 'senescence' factor is produced or reproduces itself at a slightly higher rate than the other cellular components and that it increases from one per culture to a level sufficiently high to cause death of the hyphal tips during several centimeters of growth is compatible with the experiments reported here.

\section{Variation of the number of hyphae per culture}

We mentioned earlier the hypothesis that the appearance of the 'senescence' factor in a growing mycelium is a random event. If the probability of occurrence of the 'senescence' factor in a given hyphal tip has a definite value independent of the number of hyphae per culture, then the T.R. will vary directly as the number of hyphae growing through a given transverse cross-section of the culture.

Two methods were used for varying the number of hyphae per culture: (i) mycelia were grown on corn meal agar in tubes of different cross-sectional areas; (ii) mycelia were grown in standard culture tubes on the various agar media shown in Table 2. The cross-sectional 


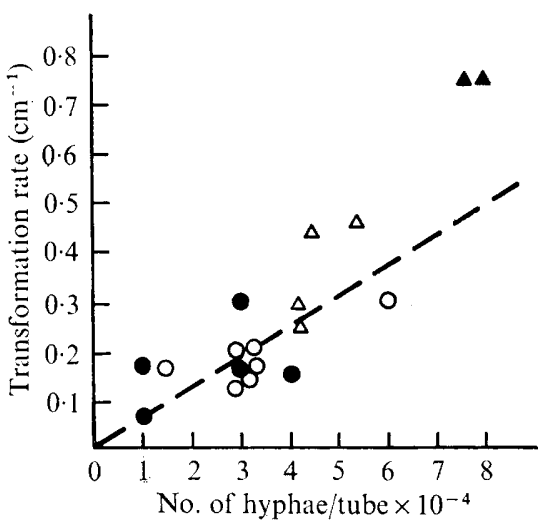

Fig. 7. Transformation rate as a function of number of growing hyphal tips per culture. of hyphae varied by varying the cross-sectional area of cultures; $\bigcirc$, various concentrations of corn meal agar (see Table 2); $\triangle$, corn meal agar supplemented with various substances (see Table 2); $\boldsymbol{\Delta}$, Florida State Minimal Media (Perham, I96I); ---, expected value of transformation rate based on value obtained for corn meal agar in standard culture tubes. Each point represents the transformation rate determined for a population of 20 mycelia.

Table 2. The hyphal density, median length of growth, transformation rate and incubation distance of mycelia of race $A$ grown on various nutrient media

\begin{tabular}{|c|c|c|c|}
\hline Media* & $\begin{array}{l}\text { Hyphal density } \\
\qquad\left(\mathrm{cm}^{-2}\right)\end{array}$ & $\begin{array}{l}\text { M.L.G. } \\
(\mathrm{cm})\end{array}$ & $\begin{array}{c}\text { T.R. } \\
\left(\mathrm{cm}^{-1}\right)\end{array}$ \\
\hline$\frac{1}{2} \times$ Corn meal agar & $2 \times 10^{4}$ & 13.0 & 0.15 \\
\hline I $\times$ Corn meal agar & $4 \times 10^{4}$ & I $4 \cdot 5$ & 0.15 \\
\hline $\mathrm{I} \times$ Corn meal agar $+0.1 \%$ ammonium acetate & $6 \times 10^{4}$ & $9 \cdot 0$ & 0.23 \\
\hline I $\times$ Corn meal agar $+0.5 \%$ ammonium acetate & $5 \times 10^{4}$ & $9 \cdot 0$ & 0.46 \\
\hline $\mathrm{I} \times$ Corn meal agar $+\mathrm{I} \cdot 0 \%$ ammonium acetate & $8 \times 10^{4}$ & $8 \cdot 0$ & 0.43 \\
\hline $\mathrm{I} \times$ Corn meal agar $+\mathrm{PO}_{4}+$ ammonium acetate $\dagger$ & $5 \times 10^{4}$ & $13 \cdot 5$ & 0.33 \\
\hline $2 \times$ Corn meal agar & $8 \times 10^{4}$ & $12 \cdot 5$ & $0 \cdot 33$ \\
\hline FSMM & $10 \times 10^{4}$ & $13 \cdot 0$ & 0.77 \\
\hline
\end{tabular}

* Each medium was tested with a population of 20 mycelia each originating from a binucleate ascospore. $\dagger 0.032 \mathrm{M}-\mathrm{Na}_{2} \mathrm{HPO}_{4}+0.008 \mathrm{M}-\mathrm{KH}_{2} \mathrm{PO}_{4}, \mathrm{pH} 7 \cdot 4$, and $0.2 \%$ ammonium acetate.

$¥$ Florida State Minimal Media (Perham, 1961).

area of the agar was varied from $0.25 \mathrm{~cm}^{2}$ (9 mm diam. tubes) to $\mathrm{I} \cdot 0 \mathrm{~cm}^{2}$ (30 mm diam. tubes). The standard culture tubes were $20 \mathrm{~mm}$ in diam. and the cross-sectional area of the media was $0.75 \mathrm{~cm}^{2}$. A germinated binucleate ascospore of race A was used to inoculate each culture tube, and at least 20 cultures were grown in each culture condition.

The T.R. is plotted in Fig. 7 as a function of number of hyphae per culture tube. The straight line represents the relationship predicted from our simple hypothesis. The values of T.R. tend to vary in the predicted manner. The values at $3 \times 10^{4}$ hyphae per tube represent standard culture tubes with the usual concentration of corn meal agar. Since each point represents only one determination of T.R. it is not possible to determine the error in this estimate. However, the standard deviation of the values of T.R. determined from a number of populations of strain A mycelia was 0.05 . If this value is applied here, $60 \%$ of the points lie within one standard deviation of the predicted value. 
Table 3. Growth of mycelia after treatment with substances that inhibit mitochondrial function

\begin{tabular}{|c|c|c|c|c|}
\hline \multirow[b]{2}{*}{ Compound* } & \multirow{2}{*}{$\begin{array}{l}\text { Duration } \\
\text { (days) }\end{array}$} & \multirow[b]{2}{*}{ Concentration } & \multicolumn{2}{|c|}{$\begin{array}{l}\text { M.L.G. after treatmen } \\
(\mathrm{cm})\end{array}$} \\
\hline & & & Treated & Controlt \\
\hline Azide & 4 & $4 \times 10^{-5} \mathrm{M}$ & $2 \cdot 0$ & $1 \cdot 0$ \\
\hline $\mathrm{KCN}$ & 4 & $4 \times \mathrm{IO}^{-3} \mathrm{M}$ & $20 \cdot 0$ & $2 \cdot 0$ \\
\hline $\mathrm{CO}$ & 3 (in dark) & $95 \%$ & $4 \cdot 0$ & $2 \cdot 5$ \\
\hline Dinitrophenol & 4 & $5 \times \mathrm{IO}^{-4} \mathrm{M}$ & $5 \cdot 0$ & $4 \cdot 0$ \\
\hline Cycloheximide & 4 & $4 \times 10^{-5} \mathrm{M}$ & I $6 \cdot 5$ & $5 \cdot 0$ \\
\hline Antimycin & 4 & Io $\mu \mathrm{g} / \mathrm{ml}$ & $5 \cdot 0$ & $6 \cdot 0$ \\
\hline Ologomycin & 4 & $\mathrm{I} \mu \mathrm{g} / \mathrm{ml}$ & $2 \cdot 0$ & $4 \cdot 5$ \\
\hline Low $\mathrm{O}_{2}$ & 4 & $\mathrm{I} \% \mathrm{O}_{2}$ & $7 \cdot 0$ & $4 \cdot 5$ \\
\hline Chloramphenicol & 4 & $2 \mathrm{mg} / \mathrm{ml}$ & $6 \cdot 5$ & 4.0 \\
\hline
\end{tabular}

* Experiments with $\mathrm{KCN}$, Azide, $\mathrm{CO}$, cycloheximide and chloramphenicol were done twice more with similar results.

$\dagger$ Average value for control cultures from each side of the $\mathrm{I} \mathrm{cm}$ section of mycelium used in each experiment.

\section{Short periods of growth under conditions which inhibit mitochondrial function}

From analogy with other cytoplasmically inherited variants in the fungi (see Esser \& Kuenen, 1967; Fincham \& Day, 197I; and Jinks, 1966, for reviews), the transformation from a 'non-senescent' to a 'senescent' mycelium might correspond to the appearance of an abnormal mitochondrion. This idea would be reinforced if mycelial growth under conditions which inhibit mitochondrial function in specific ways had a significant effect on 'senescence'. With this in mind, samples from mycelia in the early stages of the 'senescent' state were grown for 4 days in corn meal agar alone or corn meal agar media containing the drugs listed in Table 3 or in a nitrogen atmosphere containing a small percentage of oxygen or in carbon monoxide in the dark. The samples grown on unsupplemented corn meal agar were the controls for this experiment.

After 4 days, Io samples were taken from each plate and transferred to corn meal agar in standard culture tubes. The M.L.G. of each set of Io mycelia was determined. The values given in Table 3 (except for low oxygen and carbon monoxide) are for concentrations of the substances which are half those required to completely inhibit growth. At the concentrations listed in Table 3 the rate of mycelial growth was 40 to $90 \%$ less than on corn meal agar. In some cases the hyphal density was also lower than normal.

Only potassium cyanide and cycloheximide increased the M.L.G. after treatment. Either the production of the 'senescence' factor or the replication of cells containing the 'senescence' factor is inhibited by these substances. Cycloheximide inhibits protein synthesis on cytoplasmic ribosomes, but not on mitochondrial ribosomes (Lamb, Clark-Walker \& Linnane, 1968). Cyanide inhibits cytochrome $c$ oxidase (Keilin \& Hartree, 1939) but also inhibits a variety of other enzymes (Haldane, 1965). These results do not necessarily implicate abnormal mitochondria as the 'senescence' factor. They do indicate, however, that the transformation from 'senescent' to 'non-senescent' state may be promoted by much shorter term treatments than those used by Marcou (I96I). 


\section{DISCUSSION}

The experiments reported here, in conjunction with the results of Marcou (I96I), indicate that 'senescence' in both races A and S of Podospora anserina results from the appearance of a single variant entity in the cytoplasm (the 'senescence' factor). Furthermore, the rate of occurrence of the 'senescence' factor in race $\mathrm{A}$ is about Io times higher than in race $\mathrm{S}$ and the amount of growth necessary for morphological manifestation of 'senescence' in race $A$ is about $I /$ Io that in race $S$. These observations, coupled with the report of Smith \& Rubenstein (1973), indicate that the transformation rate and incubation distance are cytoplasmically inherited in races $\mathrm{S}$ and $\mathrm{A}$ and that the frequency of occurrence of the "senescence' factor and at least one of its properties (the amount of growth required for its expression) are cytoplasmically inherited.

One of the experiments reported here indicates that during the growth represented by the incubation distance the concentration of the 'senescence' factor per cell in race A increases exponentially. It seems likely that the incubation distance represents the amount of hyphal growth required for the 'senescence' factor to reach a level of concentration which inhibits growth. Although we did not determine the rate of increase of the 'senescence' factor in mycelia of race $S$, it seems reasonable that the longer incubation distance of race $S$, as compared to race A, may be the result of a lower rate of increase of concentration of the 'senescence' factor.

Marcou (I96I) suggested several models to account for 'senescence' in Podospora anserina. (i) The mutation of a cytoplasmic gene. This mutation would exert a deleterious effect on the mycelium and the relative number of mutant genes would increase as growth proceeded. (ii) An episome or provirus is released from the cellular DNA. The episome or virus in its free form would exert a deleterious effect on the mycelium and would increase in numbers as growth proceeded. (iii) A self-maintaining change occurs in some cytoplasmic structure. For example, if the structure $C$ represents the 'senescence' factor and $B$ represents the original structure, the transformation $\mathrm{B} \rightarrow \mathrm{C}$ can occur only at a low frequency when $\mathrm{C}$ is absent, but once $\mathrm{C}$ is present the rate of transformation $\mathrm{B} \rightarrow \mathrm{C}$ increases and depends on the concentration of $\mathrm{C}$. When the concentration of $\mathrm{C}$ becomes sufficiently high 'senescent' morphology results. (iv) The mycelium can exist in two or more physiological states each of which is stable. The mycelium can be converted from one state to another by a transient change in the concentration of intracellular constituents. The neighbouring hyphae would be homogeneous with respect to the concentration of these constituents. This would account for the fact that large areas of the mycelia exhibit 'senescent' morphology at the same time.

In addition to the above models, Holliday (1969) proposed that 'senescence' may result from errors in protein synthesis of the type discussed by Orgel (I963). For example, if an error occurred which altered the specificity of an RNA polymerase or an amino-acyl-tRNA synthetase molecule, errors in protein synthesis could increase exponentially and result in the death of the organism.

From Marcou's second model and the model proposed by Holliday one would expect that the nuclear genes would control the time of expression of 'senescence'. According to our data, this is not the case (Smith \& Rubenstein, 1973). If the transition from 'non-senescence' to 'senescence' were due to a transient change in concentration of intracellular constituents, as in the fourth model proposed by Marcou, the transformation rate should decrease or remain constant as the number of hyphae per culture increases. However, we have shown that the transformation rate increases linearly with the number of hyphae per culture. This 
narrows the possibilities to models I and 3 of Marcou which are consistent with the results reported here as well as previously published results. At the present time it is not possible to determine which, if either, of these models is correct. If the 'senescence' factor were a mutant cytoplasmic gene, our data indicate that the mutation rates would be approximately $\mathrm{IO}^{-7}$ and $\mathrm{IO}^{-8}$ in race $\mathrm{A}$ and $\mathrm{S}$, respectively. Also the increase in rate of production per cell division of the mutant gene would be very low; about $\mathrm{I} .02$ times normal in race $\mathrm{A}$ and $\mathrm{I} .002$ times normal in race $\mathrm{S}$. Although there is little known about mutation rates in cytoplasmic genes and differences of rates of replication between organelles carrying normal and mutant genes, these values intuitively seem acceptable.

The idea that the lifespan of an organism can be determined by the acquisition of mutant cytoplasmic genes can be extended to other organisms. However, unless the mutation occurs in most cells of the organism (this would probably require a mutation rate of about $10^{-2}$ / division) very good intercellular communication would be required to transmit the 'senescence' factor from cell to cell. In the fungi this communication can be provided by hyphal anastomosis. Although, we do not yet know to what extent intercellular communication occurs between mammalian cells, there is some evidence that macromolecular RNA can be exchanged (Kolodny, I97I). If intercellular exchange of self-replicating particles can be shown to occur between mammalian cells, then 'senescence' in Podospora anserina may provide a plausible model for the ageing of mammals.

We wish to acknowledge the excellent technical assistance of Mrs Ronate Fuchs and Mr Edward Nelbach and many helpful discussions with Drs Peter Day and Norman Giles. This work was supported by USPHS grants no. HD-040I2 and HD-5380 and NIH predoctoral fellowship no. GM 39874 (J. R. S.).

\section{REFERENCES}

Bernet, J. (1965). Mode d'action des gènes de barrage et relation entre l'imcompatibilité cellulaire et l'imcompatibilité sexuelle chez Podospora asnerina. Annales des Sciences Naturelles Botanique 6, 6I I-768.

Esser, K. \& Kuenen, R. (1967). Genetics of Fungi. New York: Springer Verlag.

Fincham, J. R. S. \& Day, P. R. (197I). Fungal Genetics, 3rd edn. Philadelphia, Pennsylvania, F. A. Davis. Haldane, J. B. S. (1965). Enzymes. Cambridge Massachusetts: The M.I.T. Press.

Holliday, R. (1969). Errors in protein synthesis and clonal senescence in fungi. Nature, London 221, I 224-I 228.

Jinks, J. L. (1966). Extrachromosomal Inheritance. Englewood Cliffs, New Jersey: Prentice Hall.

Keilin, D. \& Hartree, E. F. (1939). Cytochrome and cytochrome oxidase. Proceedings of the Royal Society B 127, $167-191$.

KolodNY, G. M. (197I). Evidence for transfer of macromolecular RNA between mammalian cells in culture. Experimental Cell Research 65, 313-324.

Lamb, A. J., Clark-Walker, G. D. \& Linnane, A. W. (1968). The biogenesis of mitochondria. 4. The differentiation of mitochondrial and cytoplasmic protein synthesizing systems in vitro by antibiotics. Biochemica et biophysica acta $\mathbf{1 6 1}, 4 \mathrm{I} 5-427$.

Marcou, D. \& Schecroun, J. (1959). La senescence chez Podospora pourrait être due a des particules cytoplasmiques infectantes. Compte rendu de l'Acad des sciences 248, 280-283.

Marcou, D. (1961). Notion de longevité et nature cytoplasmique du determinant de la senescence chez quelques champignons. Annales des sciences naturelles (Botanique series $12 e$ ) 2, 653-763.

Orgel, L. E. (1963). The maintenance of the accuracy of protein synthesis and its relevance to aging. Proceedings of the National Academy of Sciences of the United States of America 49, 517-52I.

Perham, J. E. (196I). The development of a new biochemical genetic tool: Podospora anserina Niessl. Thesis, Florida State University.

Ruzet, G. (1953a). Sur l'impossibilité d'obtenir la multiplication vegetative ininterrompru et illimitée de l'Ascomycete Podospora anserina. Compte rendu de l'Academie des sciences 237, I I06-1 I09. 
Rizet, G. (1953b). Sur la longevité des souches de Podospora anserina. Compte rendu de l'Academie des sciences $237,838-840$.

RIZET, G. (1957). Les modifications qui conduisent a la senescence chez Podospora sont-elles de nature cytoplasmique. Compte rendu de l'Académie des sciences 244, 663-665.

Rizet, G., Marcou, D. \& Schecroun, J. (1958). Deux phenomènes d'heredité cytoplasmique chez l'Ascomycete Podospora anserina. Bulletin de la Société française de physiologie végétale 4, 136-149.

SCHECROUN, J. (1962). Incompatibilité cellulaire et interactions nuclio-cytoplasmiques dans les phenomènes de barrage chez le Podospora anserina. Annales de Genetique 4, 4-50.

SMith, J. \& Rubenstein, I. (1973). Cytoplasmic inheritance of the timing of 'senescence' in Podospora anserina. Journal of General Microbiology 76, 297-304. 